\title{
Imaging Innate Immune Responses using Dual Color Stochastic Reconstruction Optical Microscopy (STORM)
}

\author{
J.S Aaron, * B. Carson* and J.A. Timlin*
}

* Bioenergy and Defense Technologies Department, Sandia National Laboratories, PO Box 5800, Albuquerque, NM 87185

Over the past decade, optical approaches have been introduced that effectively break the traditional diffraction barrier. Of particular note were the introductions of Stimulated Emission and Depletion (STED) microscopy [1], Photo-Activated Localization Microscopy (PALM) [2], and the closely related Stochastic Optical Reconstruction Microscopy (STORM) [3]. Of these, the STORM/PALM approach represents an attractive method for researchers, as it does not require highly specialized optical setups as is the case in STED, and can be more easily implemented for multi-color imaging. We have implemented a dual-color direct STORM system to simultaneously image the subresolution organization/colocalization of the TLR-4 receptor, a key mediator of innate immune response and lipopolysaccharide (LPS), a bacteria-specific antigen recognized by TLR4. We show that "super-resolution" imaging approaches such as this can provide information that is not attainable with other optical methods.

We implemented a simultaneous dual-color STORM imaging system that allows for simultaneous excitation and detection of two fluorescence channels via projection of each fluorophor's signal onto separate regions of an electron-multiplied charge-coupled device (EMCCD) (iXon, Andor Technologies). Initial characterization of our system indicates that a complete two-color data, comprising of 3000-10,000 frames, can be acquired in as little as 60 seconds due to the high sensitivity of our detector. This resulted in the absence of a need for fiduciary markers to correct for stage drift, as has been used in previous studies [3]. Furthermore, a non-linear least squares (NLLS) fit of the measured point-spread functions indicates a reliable localization accuracy of less than $40 \mathrm{~nm}$.

We have extended our studies to probe the spatial re-organization of the TLR4 receptor after stimulation with bacterially-derived LPS antigens. While distinct chemotypes of LPS have evolved among various bacteria, only certain LPS variations (such as that derived from $E$. coli) typically result in significant cellular immune response, while others (such as from the plague bacteria $Y$. pestis) do not, despite apparent binding of both to TLR-4 [4]. We are utilizing our STORM capabilities to reveal potential sub-resolution organizational differences of TLR-4 receptors in the cell membrane in response to binding of various LPS types to elucidate mechanisms of infection. In additionally, we are probing sub-resolution co-localization of the receptor with LPS. Reports have indicated that TLR4 receptors are recruited to specific, high-density membrane domains in response to LPS recognition [5]. These domains typically fall below the optical diffraction limit $(<300 \mathrm{~nm})$, thus limiting the quantitative information available from traditional fluorescence microscopy. However, using direct, independent readout of AlexaFluor 647 (Invitrogen) and Atto532 (AttoTec) labeled LPS and TLR4 receptors on the surface of P388D1 mouse macrophages (in the presence of an oxygen scavenging buffer containing mercaptoethanol [3]), the effective resolution can be increased by nearly an order of magnitude, as shown in Figure 1, far right. In Figure 2, TLR4 cluster size analysis shows that receptor aggregation in the cell membrane increases by $30 \%$ in response to 
E. coli derived LPS, a statistically significant result that is not apparent using conventional resolution microscopy.

[1] Hell, S.W.; Wichmann, J. (1994), Opt. Lett. 19 (11): 780-782.

[2] Betzig, E., et al. (2006), Science, 313(5793): 1642-1645.

[3] Rust, M.J., et al. (2006), Nat. Meth. 3, 793-796.

[4] James, C.D., et al. (2009) Biomedical Devices 11(3): 693-700.

[5] Triantafilou, M., et al., (2004) Biochem. J. 381(Pt 2): 527-536.

[6] Sandia National Laboratories is a multi-program laboratory operated by Sandia Corporation, a wholly owned subsidiary of Lockheed Martin Corporation, for the U.S. Department of Energy's National Nuclear Security Administration under contract DE-AC04-94AL85000.

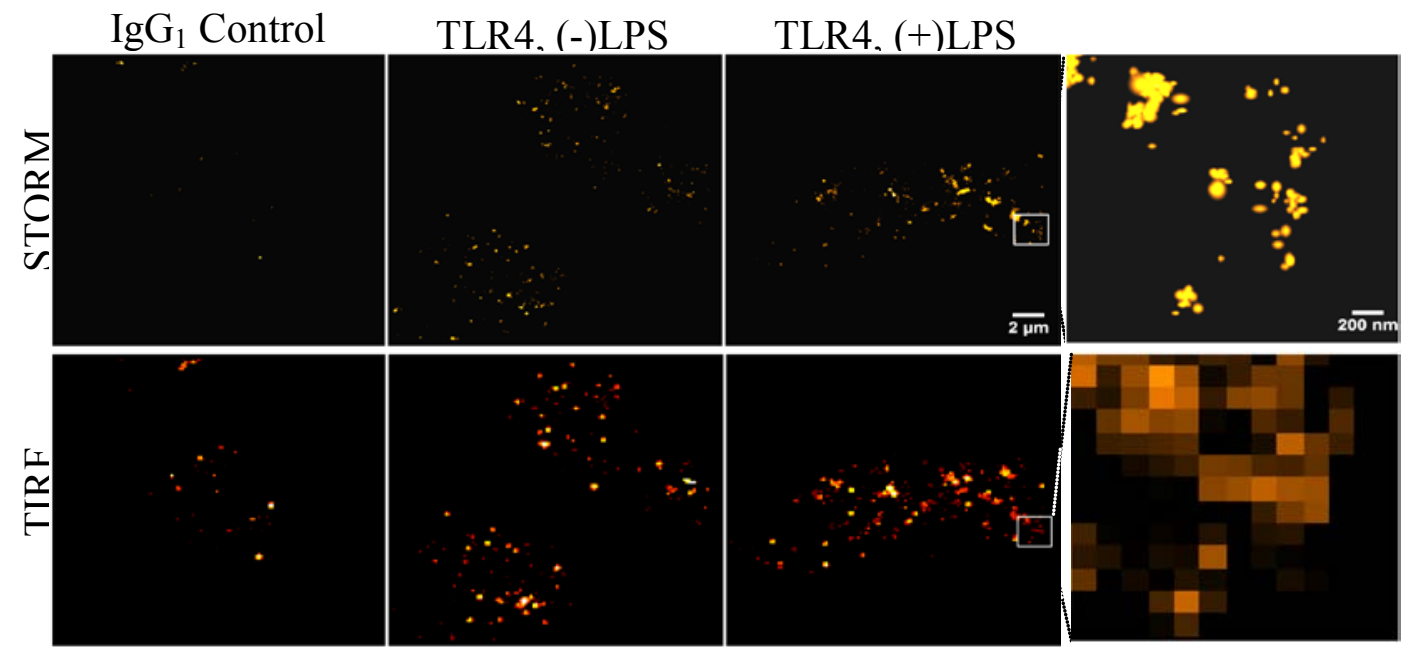

Figure 1. STORM (at top) vs. TIRF (at bottom) microscopy of TLR4 receptors in mouse macrophage cells. At left, IgG isotype controls indicate approximately $10 \%$ non-specific signal. TLR4 receptor clustering in response to LPS stimulation was measured and found to increase by $30 \%$ (see Figure 2). At far right, an image sub-region is expanded to show the large increase in available detail due to the use of STORM. The effective resolution in our STORM system was found to be $c .40 \mathrm{~nm}$.

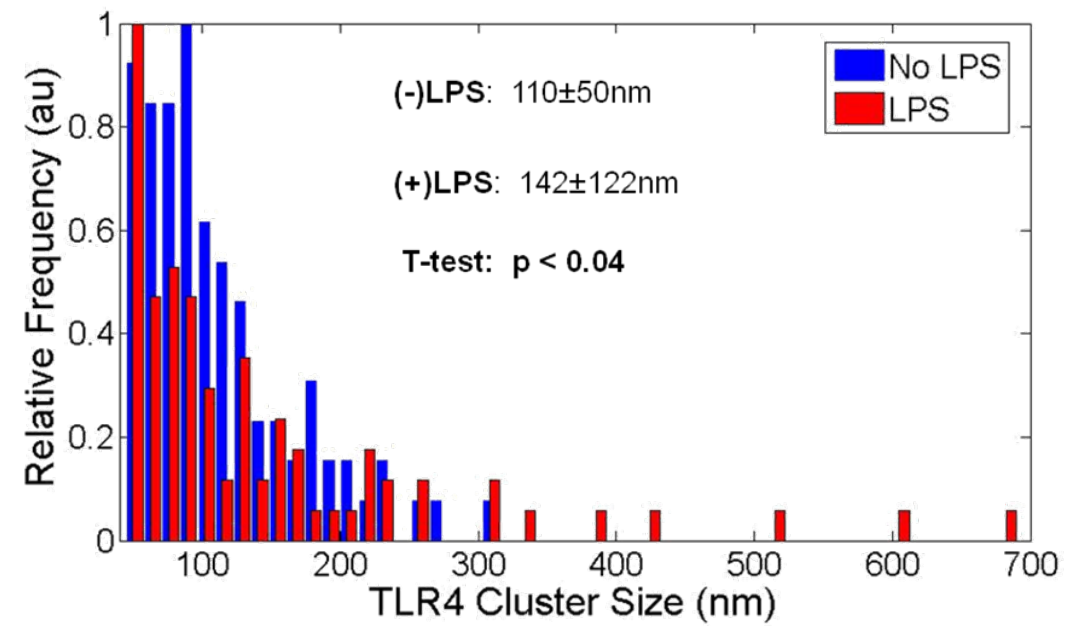

Figure 2. TLR4 cluster size analysis shows that LPS results in an average increase in receptor aggregation by $30 \%$, with particular increase in prevalence in clusters above $300 \mathrm{~nm}$ diameter. Analysis of $\mathrm{N}=\mathbf{3}$ cells suggests these changes are statistically significant $(\mathbf{p}<\mathbf{0 . 0 4})$. Interestingly, similar analysis on conventional resolution images is not able to distinguish differences in TLR4 organization between LPS-stimulated and resting cells. 